\title{
Criminologie
}

\section{Définition des gangs et identification des membres à des fins policières}

\section{Defining Street Gangs and Identifying Street Gang Members for Police Purposes \\ Definición de las pandillas callejeras y identificación de sus miembros con propósitos policiales}

\author{
Jean-Pierre Guay, Chantal Fredette, Marc-André Mercier, Nathalie Dubé, Julie \\ Hobbs, James Paixao et René-André Brisebois
}

Volume 48, numéro 2, automne 2015

Nouvelles perspectives sur le phénomène des gangs de rue

URI : https://id.erudit.org/iderudit/1033838ar

DOI : https://doi.org/10.7202/1033838ar

Aller au sommaire du numéro

Éditeur(s)

Les Presses de l’Université de Montréal

ISSN

0316-0041 (imprimé)

1492-1367 (numérique)

Découvrir la revue

Citer cet article

Guay, J.-P., Fredette, C., Mercier, M.-A., Dubé, N., Hobbs, J., Paixao, J. \& Brisebois, R.-A. (2015). Définition des gangs et identification des membres à des fins policières. Criminologie, 48(2), 83-103. https://doi.org/10.7202/1033838ar

\section{Résumé de l'article}

L'exercice qui vise à définir les gangs de rue et à en identifier les membres est complexe. Plusieurs caractéristiques de ces groupes, notamment leur caractère élusif et clandestin, leur grande hétérogénéité ainsi que leur caractère changeant nous ont poussés à en modifier notre conceptualisation. Cet article propose une définition générale et simple qui décrit le gang de rue comme un groupe criminel organisé qui se distingue des autres groupes du crime organisé définis par la loi 467.1. L'accent est toutefois mis sur le caractère opérationnel des marqueurs d'appartenance et le rationnel qui sous-tend leur développement. Une nouvelle définition des gangs jumelée à une opérationnalisation basée sur des critères observables par les organisations policières offre plusieurs avantages par rapport à une définition développée dans un cadre académique. Les implications théoriques et les enjeux pratiques sont également abordés. 


\section{Définition des gangs et identification des membres à des fins policières ${ }^{1}$}

\author{
Jean-Pierre Guay ${ }^{2}$ \\ Professeur agrégé \\ Université de Montréal \\ Institut Philippe-Pinel de Montréal \\ jean-pierre.guay@umontreal.ca

\section{Chantal Fredette \\ Enseignante, Collège Ahuntsic \\ Chargée de cours, Université de Montréal \\ chantal.fredette@collegeahunstic.qc.ca} \\ Marc-André Mercier \\ Analyste stratégique \\ Service de police de la Ville de Montréal \\ ma.mercier@spvm.qc.ca \\ Nathalie Dubé \\ Analyste stratégique \\ Service de police de la Ville de Montréal \\ nathalie.dube@spvm.qc.ca
}

\author{
Julie Hobbs \\ Sergente-détective \\ Service de police de la Ville de Montréal \\ julie.hobbs@spvm.qc.ca \\ James Paixao \\ Lieutenant-détective \\ Service de police de la Ville de Montréal \\ james.paixao@spvm.qc.ca
}

\author{
René-André Brisebois \\ Agent de planification, de programmation \\ et de recherche \\ Centre d'expertise Délinquance et troubles \\ de comportement \\ CIUSSS Centre-Sud-de-l'île-de-Montréal \\ rene-andre.brisebois@cjm-iu.qc.ca
}

RÉSUMÉ - L'exercice qui vise à définir les gangs de rue et à en identifier les membres est complexe. Plusieurs caractéristiques de ces groupes, notamment leur caractère élusif et clandestin, leur grande hétérogénéité ainsi que leur caractère changeant nous ont poussés à en modifier notre conceptualisation. Cet article propose une définition générale et simple qui décrit le gang de rue comme un groupe criminel organisé qui se distingue des autres groupes du crime organisé définis par la loi 467.1. L'accent est toutefois mis sur le caractère opérationnel des marqueurs d'appartenance et le rationnel

1. Les auteurs sont membres d'un comité de travail sur la définition des gangs et sur l'identification des membres. Ce comité est formé de représentants du Service de police de la Ville de Montréal, du Service du renseignement criminel du Québec, du Centre d'expertise Délinquance et troubles de comportement du Centre intégré universitaire de santé et de services sociaux du Centre-Sud-de-l'île-de-Montréal et de chercheurs du Centre international de criminologie comparée de l'Université de Montréal.

2. École de criminologie et Centre international de criminologie comparée, Université de Montréal, C. P. 6128, succ. Centre-ville, Montréal (Québec), Canada, H3C 3J7. 
qui sous-tend leur développement. Une nouvelle définition des gangs jumelée à une opérationnalisation basée sur des critères observables par les organisations policières offre plusieurs avantages par rapport à une définition développée dans un cadre académique. Les implications théoriques et les enjeux pratiques sont également abordés.

MOTS CLÉs - Gangs de rue, définition, identification, crime organisé, police.

\section{Introduction}

Les groupes criminels urbains nommés "gangs de rue» font partie intégrante du paysage médiatique nord-américain. Dans de grandes villes comme Los Angeles, Chicago, Philadelphie ou Montréal, rares sont les semaines où ces groupes ne font pas la manchette. Cette visibilité et le type de crimes exposés par les médias contribuent, en partie du moins, à accroître le sentiment d'insécurité des citoyens, ce qui les pousse à exercer une importante pression sur les organisations responsables de la répression. Les réactions des autorités ont rapidement fait comprendre à la communauté que le problème des gangs de rue doit être pris au sérieux (Katz et Webb, 2006). De plus, de tels efforts se soldent généralement par un afflux de personnes accusées devant les tribunaux et un nombre accru de personnes placées sous la responsabilité des organismes de prise en charge sociale et pénale. Une telle situation a pour effet de provoquer une série de conséquences en cascade sur l'appareil de justice et sur les délinquants eux-mêmes.

Les motifs qui poussent les autorités à porter une attention particulière aux gangs de rue sont que les membres de ces groupes sont responsables d'une quantité importante de délits (Decker et Pyrooz, 2010; Esbensen et Huizinga, 1993). Cette délinquance accrue fait en sorte que les membres de gangs sont plus surveillés et les quartiers où opèrent ces groupes font l'objet d'une attention toute particulière des autorités policières (Bureau of Justice Assistance, 1999). Une fois judiciarisés, les membres de gangs font aussi l'objet de préoccupations particulières de la part des juges et des procureurs. En effet, le fait d'être identifié comme membre de gangs augmente la probabilité d'être reconnu coupable (Eisen, 2009), et mène parfois à une peine plus sévère (Miethe et McCorkle, 2006). Une fois placés sous la responsabilité du système de justice pénale, les délinquants associés aux gangs risquent de faire l'objet d'une surveillance particulière de la part des intervenants. Ils seront fort possiblement jugés à plus haut risque de récidive (Guay, 2012) compte tenu de leur unique affiliation aux gangs de rue et pour- 
raient faire l'objet d'une prise en charge plus sécuritaire en raison de leur implication accrue dans les incidents disciplinaires et de violence (DeLisi, 2003; Gaes, Wallace, Gillman, Klein-Saffran et Suppa, 2002). Ultimement, ils se verront octroyer moins facilement des mesures d'élargissement de la peine, et briseront plus fréquemment les conditions de leur libération conditionnelle (McShane, Williams et Dolny, 2003). En somme, le fait d'appartenir aux gangs ou d'être identifié comme un membre de gang a des conséquences importantes tant sur l'appareil pénal que sur la personne contrevenante elle-même.

Ces constats mettent en perspective toute la pertinence de bien identifier les délinquants liés aux gangs de rue. Cet article propose deux parties. Une première portera sur la question des définitions des gangs et fera état de l'utilité d'une définition commune. Elle abordera ensuite les principales difficultés entourant l'étude des gangs de rue. Finalement, elle présentera les principaux critères utilisés pour définir les gangs de même que la définition la plus communément utilisée au Québec. Une deuxième partie portera sur la proposition d'une nouvelle définition des gangs. Elle débutera par une présentation sommaire du modèle multidimensionnel de Guay et Fredette (2010) sur lequel elle s'appuie. Elle décrira la définition retenue, les critères d'identification des membres de même que la méthodologie préconisée pour en tester la fidélité et la validité. L'article se terminera par quelques éléments de réflexion sur l'application d'une telle stratégie ainsi que sur le processus de désaffiliation.

\section{La définition des gangs de rue}

La prochaine section comporte trois sous-sections distinctes, soit l'utilité d'une définition commune, les principaux défis et enjeux rencontrés dans la définition des gangs de rue, et les principaux critères généralement utilisés pour définir les gangs.

Depuis l'apparition des premières définitions du phénomène, notamment celle de Thrasher (1927), plusieurs dizaines d'autres ont été proposées, discutées et critiquées. Un des rares consensus concernant la question des gangs de rue est qu'il est difficile d'en arriver à une unanimité quant à la façon de définir ces groupes, et qu'il n'existe pas de méthode commune pour identifier les délinquants qui y sont associés (Esbensen, Winfree, He et Taylor, 2001 ; Katz, 2003 ; Klein et Maxson, 2006; Wortley, 2010). Wortley (2010) souligne que la façon de conce- 
voir les définitions de gangs est fonction du contexte dans lequel elles seront utilisées. En effet, les définitions proposées par les acteurs du système de justice ont généralement tendance à être différentes de celles proposées par les chercheurs du milieu universitaire (Wortley, 2010). Alors que ces derniers souhaitent proposer une définition qui les guidera dans l'étude du phénomène social, les praticiens de la justice opteraient plutôt pour une définition utile dans l'atteinte de leurs objectifs respectifs, une définition qui permettra l'identification des personnes qui en font partie. La présente démarche s'inscrit dans la seconde perspective.

\section{L'utilité d'une définition commune des gangs}

Wortley (2010) propose une synthèse des bénéfices d'une définition commune des gangs. Selon lui, une définition commune: 1) favorise des estimations exactes des activités liées aux gangs à l'échelle nationale, provinciale et municipale; 2) renforce notre capacité d'établir des comparaisons régionales; 3 ) permet de documenter les facteurs de risque associés aux activités liées aux gangs dans des administrations précises ; 4) est utile pour documenter le montant du financement public requis pour s'attaquer aux problèmes liés aux gangs dans des administrations précises; 5) habilite les responsables de l'application de la loi des différentes administrations à communiquer dans la même «langue» et à acquérir une compréhension commune des gangs, des membres de gang et des actes criminels liés à un gang; et 6) rehausse la qualité des enquêtes sur les gangs auxquelles participent les services de police de différentes régions (Wortley, 2010, p. 3-4). À cela on pourrait ajouter qu'une bonne définition opérationnelle pourrait permettre d'estimer l'effet sur ces groupes des efforts de prévention et de répression.

\section{Les difficultés associées à l'étude des gangs de rue}

Dans de nombreux travaux de recherche sur les gangs, le moyen le plus fréquemment utilisé pour identifier les membres est l'auto-identification. Le fait est qu'une proportion non négligeable des connaissances sur les membres de gangs sont issues de sondages auprès de populations de jeunes (Egley et Howell, 2013; Gatti, Tremblay, Vitaro et McDuff, 2005; Lanctôt et LeBlanc, 1997; Thornberry, Krohn, Lizotte, Smith et Tobin, 2003) ou de recherches qualitatives (Charland, 2010; Cousineau, Hamel et Fournier, 2005; Decker et Van Winkle, 1996; 
Demers, 2015; Vigil, 1988). Tout jeune s'identifiant à un gang est jugé comme en faisant partie, peu importe ses conceptions de ce qu'est un gang ou sa réelle participation aux activités d'un tel groupe. Parmi les conséquences de telles stratégies, on retrouve le fait que le nombre de membres de gangs peut apparaitre gonflé, surreprésenté. Bien qu'elle comporte un certain nombre d'avantages, la stratégie fondée sur l'autoidentification demande un regard de l'intérieur. Si l'auto-identification est intéressante en contexte de recherche, elle est pour ainsi dire peu utile dans un contexte comme celui des acteurs du système de justice comme la police ou toute organisation de prise en charge pénale. Sans surprise, les personnes contrevenantes n'ont que très peu d'avantages à révéler leur affiliation à un groupe comme les gangs de rue, ce qui fait en sorte qu'on peut difficilement s'y fier.

Les gangs sont difficiles à étudier pour de nombreuses raisons. Une première concerne le caractère élusif et clandestin des activités des membres du groupe. En effet, l'observateur n'a généralement qu'une lecture extérieure du problème. Nombreux sont toutefois les travaux de recherche misant sur une stratégie d'observation participante qui ont permis d'avoir une lecture fine du fonctionnement de différents gangs au fil des années. Néanmoins, une telle stratégie comporte un certain nombre de limites bien documentées, parmi elles, celles relatives à la fidélité et à la généralisabilité sont fréquemment évoquées (Dewalt et Dewalt, 2002).

Une seconde raison concerne la très grande hétérogénéité de ces groupes. Il n'y a pas, pour ainsi dire, deux gangs semblables (Thrasher, 1927). Par conséquent, il est difficile de parler des gangs comme d'un seul et même ensemble. Beaucoup de travaux ont été effectués sur les différents types de gangs. Dans le champ des gangs de rue, plusieurs chercheurs en sont venus à proposer des systèmes classificatoires, généralement des typologies théoriques (des prototypes) de gangs (voir notamment Huff, 1989; Klein et Maxson, 2006; Sanchez-Jankowski, 1991; Thrasher, 1927) et des typologies théoriques de membres de gangs (voir notamment Spergel, 1995; Vigil, 1988; Yablonsky, 1970). Bien qu'ils présentent un intérêt certain, plusieurs de ces systèmes classificatoires souffrent de problèmes de fidélité, attribuables en bonne partie à l'absence de règles d'attribution.

Une troisième caractéristique importante est liée au fait que les groupes criminels comme les gangs de rue sont des groupes changeants. Les membres vont et viennent constamment; en moyenne, ils 
participent à ces groupes durant une à deux années (Krohn et Thornberry, 2008; Melde et Esbensen, 2011; Weerman, Lovegrove et Thornberry, 2015). Certains groupes meurent aussi vite qu'ils sont nés, tandis que d'autres perdurent et s'adaptent à leur environnement, survivant malgré tout au départ des uns et des autres. Certains membres se joignent au groupe, le quittent définitivement ou temporairement, alors que d'autres sont incarcérés ou éloignés des activités du groupe par une mesure pénale. Leur emplacement géographique est lui aussi parfois changeant. Alors que certains groupes s'identifient spécifiquement à un secteur, une ligne d'autobus ou même une rue, d'autres s'étendent, se fusionnent en plus grands groupes, changent de nom ou se scindent en différents sous-groupes, eux aussi souvent formés de différentes petites cliques.

\section{Critères couramment utilisés pour définir les gangs}

Plusieurs chercheurs et praticiens ont néanmoins proposé différentes définitions des gangs. Une bonne définition, du moins du point de vue des praticiens, devrait décrire de manière utile le phénomène, c'est-àdire qu'elle devrait permettre de prendre de bonnes décisions. Les critères, quant à eux, devraient permettre d'identifier correctement les membres, tout en évitant la suridentification et la sous-identification. Des critères trop vagues ou trop inclusifs font en sorte que trop de personnes sont identifiées comme appartenant aux gangs. Les conséquences sont alors nombreuses. Par exemple, ils ont tendance à donner l'impression que le phénomène des gangs est plus présent qu'il ne l'est réellement, en plus de faire en sorte que des personnes n'appartenant pas réellement à de tels groupes se voient identifiées comme membres, avec les conséquences mentionnées plus haut. À l'inverse, une définition sous-inclusive fait en sorte de minimiser la présence des gangs et le risque posé par leurs membres, et cette sous-identification mène à une sous-allocation de ressources pour faire face au problème. En somme, il n'existe pas en soi de définitions parfaites. On jugera de la pertinence de la définition des gangs par sa capacité à répondre aux objectifs de ses concepteurs et de ses utilisateurs. Dans le cadre du développement d'une définition et de critères pour les organisations de prise en charge pénale, on préférera les définitions qui permettent d'identifier les auteurs d'infraction qui causent le plus de tort à la société par les crimes qu'ils commettent. 
Dans une revue éclairante des principaux travaux sur les définitions de gangs, Wortley (2010) recense au moins une douzaine de principaux critères utilisés pour définir les gangs de rue: l'âge des membres du groupe, un nom ou une identité, des signes et des symboles distinctifs, le contrôle ou la revendication d'un territoire, des aspects liés au niveau d'organisation du groupe, un certain nombre de membres, une certaine durabilité ou stabilité, certains codes ou rituels, une préférence pour une criminalité urbaine, une participation régulière ou continue du groupe à des actes criminels, violents ou délinquants ainsi qu'une certaine origine ethnique ou raciale commune. Ces caractéristiques se retrouvent généralement dans la majorité des définitions des gangs recensées dans la littérature scientifique.

\section{La définition policière actuelle des gangs de rue au Québec}

Au Québec, une des définitions qui a été la plus fréquemment utilisée est une définition policière, à savoir celle proposée par le Service de renseignement criminel du Québec (SRCQ) et le Service de police de la Ville de Montréal (SPVM):

Le gang de rue est un regroupement plus ou moins structuré d'adolescents et de jeunes adultes qui privilégient la force et l'intimidation du groupe pour accomplir des actes criminels, et ce, dans le but d'obtenir pouvoir et reconnaissance ou de contrôler des sphères d'activités lucratives ${ }^{3}$.

Au regard de critères proposés par Klein et Maxson (2006), une telle définition, si utile soit-elle pour les autorités policières et gouvernementales, pose un certain nombre de problèmes. En effet, l'inclusion de différents éléments descripteurs (qui décrivent plus qu'ils ne permettent de définir) tels que le but allégué et les principaux moyens privilégiés fait en sorte qu'elle peut dans certains cas s'avérer tantôt surinclusive (trop de gens sont considérés comme membres), tantôt sous-inclusive (trop peu de gens sont considérés comme membres). Un groupe répondant à l'ensemble des critères mais sans réelle structure claire pourrait difficilement dans ce contexte être qualifié de gang de rue, alors qu'un groupe formé spontanément ou éphémère le pourrait. Si les constituants de cette définition ne tiennent pas compte des propositions de Klein et Maxson (2006), ils posent aussi un certain nombre de difficultés rela-

3. www.spvm.qc.ca/fr/documentation/publications-actualites-gangs-de-rue.asp 
tives à l'utilisation des critères et à leur fidélité. En effet, certains d'entre eux sont libellés de manière plutôt floue, on n'a qu'à penser à « regroupement plus ou moins structuré», ou au fait qu'il est particulièrement complexe d'inférer la motivation sous-jacente aux activités d'un groupe. Finalement, le gang semble n'être réduit qu'à la simple finalité instrumentale de commettre des crimes, ce qui pourrait être largement remis en question.

\section{Le développement d'une nouvelle définition des gangs pour les organisations policières}

La prochaine section présente le fruit du travail de développement d'une définition des gangs de rue proposée par un comité de travail conjoint réunissant des professionnels du Service de police de la Ville de Montréal, du Service de renseignement criminel du Québec, du Centre d'expertise Délinquance et troubles de comportement du Centre intégré universitaire de santé et de services sociaux du Centre-Sud-de-l'Île-deMontréal et des chercheurs du Centre international de criminologie comparée de l'Université de Montréal. Une telle définition devrait permettre de dénombrer les membres sur le territoire de Montréal de manière cohérente, de servir à des fins de renseignement et, dans de rares exceptions, de servir de base à la preuve de gang lors de témoignages à la cour. Elle visera à proposer des critères opérationnels observables du point de vue des organisations policières, ce qui fait parfois défaut aux définitions universitaires. Cette définition s'inspire du modèle multidimensionnel des gangs (Guay et Fredette, 2010), lequel est brièvement présenté dans la section suivante.

Un cadre pour mesurer l'appartenance aux gangs: le modèle multidimensionnel de Guay et Fredette (2010)

La définition des gangs et plus spécifiquement l'identification des membres de gangs est un exercice difficile. Une large part de ces difficultés est liée à la nature même du phénomène. Les stratégies pour comprendre, mesurer et étudier les propriétés du groupe (le gang) et les caractéristiques des unités qui le composent (les membres de gangs) ont été récemment reconsidérées dans une série de travaux québécois sur la mesure du phénomène des gangs (Guay et Fredette, 2010; Guay, Fredette et Dubois, 2014; Guay et Gaumont-Casias, 2009). 
Jusqu'à présent, pour des raisons de simplicité, la notion de gang de rue a été traitée comme un état discret, c'est-à-dire une entité concrète aux qualités qui la distinguent des autres. Lorsque les gangs sont considérés comme des entités discrètes (ils sont perçus comme fondamentalement différents des autres groupes criminels), l'exercice d'identification des membres est très similaire à celui du diagnostic médical. Il s'agit alors d'identifier les marqueurs les plus saillants et les indicateurs les plus précis de l'appartenance aux gangs (comme les six critères d'identification actuellement utilisés par le SPVM). Or, contrairement au diagnostic de certaines maladies, il n'existe pas de signes dits pathognomoniques, à savoir des marqueurs qui témoignent par leur seule présence de la maladie et qui permettent d'établir un diagnostic certain. Aucun signe observable ne témoigne à lui seul de l'appartenance aux gangs. Afin de déterminer si une personne appartient aux gangs, il s'agit plutôt d'identifier différents indicateurs qui, à eux seuls, ne peuvent garantir l'appartenance (utiles mais non suffisants) mais qui, en combinaison, le peuvent. Or, une telle logique nécessite une opérationnalisation valide et fidèle des critères. On doit s'assurer que les signes sélectionnés témoignent bel et bien de l'association (validité), et qu'ils sont bien mesurés (fidélité). En présence de problèmes de validité ou de fidélité, les risques de suridentification et de sous-identification sont par conséquent accrus. En bref, les gangs et les membres de gangs seraient des unités présentant une configuration particulière de caractéristiques sur des ensembles multidimensionnels de traits latents. Les gangs seraient donc des groupes aux frontières floues et changeantes, dont les membres partagent un certain nombre de caractéristiques entre eux, mais aussi avec d'autres membres de groupes similaires, comme les bandes de jeunes ou les motards, et le crime organisé italien dit traditionnel.

Dans la perspective d'étudier et de paramétrer les caractéristiques des groupes criminels comme les gangs de rue et leurs membres, Guay et Fredette (2010) ont proposé de documenter les différentes dimensions utiles à leur compréhension. Le développement de ce qui allait devenir le modèle multidimensionnel des gangs reposait sur deux sources de données: l'analyse des principales typologies de membres de gangs (les sous-types, les critères de classification utilisés, etc.) et les travaux issus de la littérature scientifique sur les caractéristiques des membres de gangs. Les résultats de l'analyse de la littérature furent par ailleurs présentés aux experts dans le cadre de groupes de discussion. 


\section{Groupes de discussion}

Le fruit du travail de recension de la littérature a été abordé dans le cadre de groupes de discussion d'experts et de cliniciens spécialisés dans l'intervention auprès des gangs. L'objectif de cette démarche de consultation auprès d'experts consistait principalement à explorer la validité de contenu (Anastasi, 1988) des dimensions relevées dans la littérature scientifique, et l'applicabilité des notions émanant principalement de la littérature américaine sur le phénomène (Lynn, 1986). À la lumière des travaux de Lawshe (1975), les dimensions devaient refléter l'essentiel des contenus jugés utiles pour comprendre l'appartenance.

Dans le cadre des groupes de discussion qui se sont déroulés en 2007, une dizaine d'intervenants issus de différents milieux de la pratique après des gangs de rue ont été sollicités pour leur connaissance de la problématique. Parmi eux, on retrouvait des professionnels des centres jeunesse, des policiers spécialisés dans la question des gangs, des intervenants des services correctionnels du Québec, des procureurs ainsi que des agents des services frontaliers. Au total, trois rencontres de deux heures réunissaient des groupes de six à huit participants à la fois. L'objectif de la discussion était de connaitre les éléments à considérer pour identifier les membres de gangs de rue. Les questions préalablement envoyées aux participants étaient les suivantes: "Quels sont les aspects à prendre en compte pour apprécier le niveau d'engagement et d'implication d'une personne dans les gangs? Qu'apprend-on à propos d'une personne si on sait qu'elle appartient aux gangs?»

Au total, au moins six thèmes différents sont ressortis des discussions. L'analyse des données a révélé que pour bien comprendre les membres de gangs, il était utile d'examiner au moins quatre thèmes principaux: 1) l'adhésion de la personne à la culture du gang; 2) la participation aux activités criminelles du groupe; 3) la présence de traits psychopathiques (ou d'un style de fonctionnement impulsif et coercitif); et 4) la place occupée dans le réseau et la structure du gang. Parmi ces quatre thèmes, trois concernent des comportements ou des manifestations comportementales visibles (manifestations de l'adhésion à la culture, activités criminelles et la place dans le réseau) tandis que la présence de traits psychopathiques concerne une caractéristique psychologique, plus difficilement observable sans un examen approfondi. Deux thèmes secondaires ont été évoqués par les participants, mais les discussions les ont amenés à ne pas les retenir ou à les intégrer aux 
quatre principaux : 5) l'utilisation de la violence, de l'intimidation et de la coercition; et 6) le désengagement des structures de contrôle social. Dans le cas de l'utilisation de la violence, les participants soulevaient que les membres de gangs étaient à même d'avoir recours à une violence instrumentale pour s'attirer le respect des pairs. Elle peut être motivée par différents facteurs comme le besoin de pouvoir ou de leadership ou pour convaincre de sa valeur. Cette notion a par ailleurs été intégrée au fil des discussions à l'idée que les membres de gangs partagent un certain nombre de traits psychopathiques, lesquels sont souvent caractérisés par une utilisation de la violence instrumentale. La notion de désengagement des structures de contrôle social a pour sa part fait l'objet d'un certain nombre de discussions. Pour les intervenants, principalement ceux provenant des organisations responsables du suivi de ces personnes (intervenants des centres jeunesse et du service correctionnel du Québec), les personnes impliquées dans les gangs étaient caractérisées par un faible niveau d'attachement envers leur communauté, provenaient de quartiers fortement désorganisés, avaient un faible attachement à leurs parents et à l'école, et peu d'aspirations positives futures. Cet aspect n'a toutefois pas été retenu en raison du fait qu'il constituait un élément développemental mais non définitionnel des gangs.

\section{La définition policière des gangs}

Dans le contexte policier canadien, la définition des gangs de rue est nichée dans la définition du crime organisé. Au regard des organisations policières, les gangs de rue constituent une variation du crime organisé, au même titre que les motards ou la mafia italienne. Il est convenu dans le projet MINERVE 4 que les gangs de rue sont une manifestation des organisations criminelles telles que définies à l'article 467.1 (1) du Code criminel. Les souches du crime organisé représentent des sous-catégories de ce concept très large et inclusif qu'est celui de l'organisation criminelle. L'organisation criminelle est définie comme suit:

4. Le projet MINERVE est un protocole d'entente visant à faire le partage de renseignements stratégiques entre les trois grands corps policiers au Québec, le SPVM, la SQ et la GRC. Il optimise la collecte et la diffusion des renseignements qui servent de base à la contribution québécoise aux évaluations nationales de la menace que constitue le crime organisé au pays, lesquelles sont sous la responsabilité du Service canadien de renseignements criminels (SCRC). 
Groupe, quel qu'en soit le mode d'organisation: a) composé d'au moins trois personnes se trouvant au Canada ou à l'étranger; b) dont un des objets principaux ou une des activités principales est de commettre ou de faciliter une ou plusieurs infractions graves qui, si elles étaient commises, pourraient lui procurer - ou procurer à une personne qui en fait partie -, directement ou indirectement, un avantage matériel, notamment financier. La présente définition ne vise pas le groupe d'individus formé au hasard pour la perpétration immédiate d'une seule infraction.

À la suite des travaux du comité, une définition simple a été proposée afin de distinguer le gang de rue des autres organisations criminelles.

Le gang de rue est une organisation criminelle qui se distingue par une culture spécifique et une structure décentralisée.

L'utilisation d'une telle définition nécessite donc de documenter trois grands domaines relevés dans le modèle multidimensionnel: les activités criminelles, les éléments de culture de gangs et la place occupée dans la structure du groupe ${ }^{5}$.

La première composante de la définition concerne les activités criminelles des gangs. Ces groupes s'adonnent principalement à une criminalité de trafic de stupéfiants, mais aussi à toute une variété d'activités criminelles, dont celles liées à la prostitution et à divers crimes de violence et d'intimidation.

La seconde composante concerne la culture particulière de ces groupes. En effet, on attribue généralement à la culture de gangs l'accélération et l'aggravation des conduites délinquantes des personnes qui fréquentent ces groupes (Cureton, 2002; Decker, 1996; Fredette, 2014; Hagedorn, 2008; Hughes et Short, 2005; Klein, 1995; Sanders, 1994; Thornberry et al., 2003). Dans le cadre de ses travaux sur la culture de gang, Fredette (2014) rappelle que les membres partagent généralement un certain nombre de signes et de symboles, en plus d'adhérer à différents rituels, règles, normes et valeurs. Ils préconisent dans une large mesure les manifestations de leur groupe, portent fièrement des signes et des symboles de leur groupe (Sachs, 1997; Savelli, 2004), et étalent leur richesse (Hagedorn, 2008). Certains ont développé des codes non verbaux et utilisent différents signes dans leurs communications (Landre, Miller et Porter, 1997). Les gangs de rue

5. Même si la littérature scientifique laisse croire que les membres de gangs partagent un certain nombre de caractéristiques attribuables à un style de personnalité psychopathique ou antisocial, cette composante n'a pas été incluse dans les domaines investigués, faute d'information de qualité dans les données accessibles. 
recourent également à différentes règles et rituels dans leur vie de groupe. Les personnes qui honorent les règles du groupe sont respectées (Decker et Van Winkle, 1996; Sanders, 1994) et il n'est pas rare de voir des rites initiatiques (Vigil, 1988). Finalement, les gangs ont tendance à partager un certain nombre de normes et de valeurs, comme la suprématie masculine, la loyauté et la cohésion de groupe (Cohen, 1955; Hagedorn, 2008). Ils adoptent également des attitudes misogynes dans lesquelles l'expression brute de la masculinité stéréotypée est valorisée (Corriveau, 2009; Dorais et Corriveau, 2006; Totten, 2000). Ils n'ont d'ailleurs pas peur de s'afficher publiquement (Felson, 2014) et n'hésitent pas à intimider les acteurs du système de justice.

La troisième composante de la définition à documenter afin d'identifier les membres concerne la place occupée dans la structure criminelle du groupe. Les gangs sont souvent décrits comme des entités aux structures et leadership informels. Ils sont souvent dépeints comme des conglomérats de petites cliques, des groupes plus ou moins disparates autour desquels gravitent différents délinquants impliqués à divers degrés dans des activités criminelles et antisociales (McGloin, 2005; Papachristos, 2006; Sarnecki et Petterson, 2001). Contrairement à certains autres groupes criminels dans lesquels les règles d'adhésion et les structures décisionnelles sont relativement claires, on n'a qu'à penser aux motards criminels, le fonctionnement des gangs est plus informel et leur structure plus plastique. Un processus d'identification des membres ne pourrait être complet sans une mesure de la place occupée par les personnes dans le réseau criminel du gang.

\section{Les critères d'identification et le développement de mesures}

Lorsque le centre d'intérêt est la personne contrevenante, comme c'est le cas dans le travail des organisations de prise en charge pénale comme la police, la définition du gang de rue n'est que d'une utilité limitée. En effet, il est nécessaire d'avoir accès à des critères d'attribution qui vont permettre à l'observateur de déterminer qui fait ou non partie d'un gang. Or, ce processus d'identification pose un certain nombre de problèmes, notamment en raison des questions de fidélité des critères utilisés. Il est donc nécessaire d'augmenter le nombre d'indicateurs afin d'avoir une vision plus juste de l'implication.

L'objectif de la présente démarche était donc de trouver un certain nombre d'indicateurs imparfaits des trois dimensions observables à 
l'aide des données policières. La stratégie préconisée a été celle du développement d'échelles, en partie fondée sur les travaux de Wilson (2005). La première étape a consisté à concevoir une grille préliminaire d'indicateurs des trois dimensions. Ces items ont fait l'objet d'une première codification sur la base des données accessibles dans les bases de données policières concernant une vingtaine de dossiers. Des ajustements ont été apportés à la grille, principalement afin de préciser certains items ou d'en éliminer certains difficilement codifiables. La deuxième étape sera de mettre à l'épreuve la fidélité des différents items au moyen d'un protocole d'accord interjuge, dans lequel une trentaine de dossiers de personnes contrevenantes seront codifiés par deux auxiliaires de recherche préalablement formés. Ultimement, les propriétés métriques d'une telle structure seront étudiées à l'aide d'une série d'analyses de Rasch (Bond et Fox, 2001) et à l'aide de la théorie de la réponse à l'item (TRI) à deux paramètres (de Ayala, 2009), lorsque le nombre de dossiers codifiés sera atteint. Les items les plus fidèles et les plus discriminants seront retenus afin de concevoir différentes mesures dimensionnelles des trois concepts retenus. Par ailleurs, certains items occuperont une place plus importante dans la mesure de chacune des dimensions. La stratégie de construction d'échelle basée sur les modèles à variables latentes comme la TRI offre de nombreux avantages, comme celui d'attribuer moins de poids aux items moins discriminants et moins graves (comme des items potentiellement descripteurs), et plus à d'autres (comme les items définitionnels). L'unidimensionnalité des concepts sera testée à l'aide d'analyses factorielles. Ultimement, les dimensions serviront à déterminer des seuils au-delà desquels l'appartenance ne fait plus de doute, qui seront définis sur la base de l'analyse des données. Ces dimensions permettront aussi éventuellement de documenter les configurations de paramètres les plus courantes, à savoir les prototypes de membres de gangs les plus courants. Au besoin, il serait possible de créer différentes typologies empiriques de membres.

\section{Les activités criminelles}

La dimension des activités criminelles a été étudiée à l'aide d'un total des différents crimes commis par la personne. L'accent a été mis sur les crimes de marché (trafic de stupéfiants et d'armes, proxénétisme, traite de personnes, tenue de maison de jeu ou de paris), les crimes contre la personne (homicide, tentative de meurtre, complot en vue de commettre 
un meurtre, agression sexuelle, vol qualifié, voies de fait, séquestration ou enlèvement, extorsion, incendie criminel, intimidation ou menaces) et certains crimes acquisitifs (fraude, vol, introduction par effraction, recel, contrefaçon). Le but était de mesurer les différents paramètres de la carrière criminelle des personnes et à quel point celles-ci participaient aux activités criminelles souvent pratiquées par les gangs.

\section{L'adhésion à la culture de gang}

La dimension de l'adhésion à la culture de gang a été grandement inspirée des travaux de Fredette (2014) et a été mesurée elle aussi à l'aide de différents indicateurs. Parmi eux, on retrouve le port de vêtements ou de tatouages à l'effigie du gang, l'aveu d'appartenance à ces groupes sur différents médias sociaux, différents propos et aveux publics tenus auprès de policiers, la participation à des manifestations de solidarité ou des événements commémorant le décès d'un ancien membre, etc.

La place occupée dans le réseau criminel

Finalement, la question de la place occupée dans le réseau criminel a été mesurée à son tour à l'aide de différents indicateurs. Parmi eux, on retrouve la participation à des activités criminelles en groupe, des liens étroits avec différentes personnes associées aux gangs, des liens d'intermédiaires avec d'autres groupes criminels, etc. Différents paramètres d'analyse de réseau seront également utilisés pour caractériser les liens de chacune des personnes, entre autres pour comprendre leur fonctionnement dans ces groupes à l'aide de mesures de centralité et de centralité d'intermédiarité (Morselli, 2009).

\section{L'application d'un outil d'identification et la nécessité des critères de désaffiliation}

Afin de s'assurer d'une utilisation adéquate d'un tel outil, il sera toutefois souhaitable de préconiser une formation pour les utilisateurs, généralement des analystes et des policiers spécialisés. Comme pour d'autres instruments du même type, une telle formation pourrait nécessiter une mise à plat des principes théoriques et pratiques qui régissent l'établissement de tels critères, l'explication des règles de cotation de chacun des items, ainsi que des exercices afin de bien s'approprier le 
fonctionnement de l'outil. Ultimement, les personnes accréditées à utiliser de tels critères seraient autorisées à effectuer l'analyse et à statuer sur l'appartenance de chacune des personnes visées par la procédure à l'aide d'une structure formelle. Cela permettra, entre autres, d'uniformiser les pratiques d'identification, de formaliser la démarche et de forcer les utilisateurs à documenter leurs décisions de manière adéquate pour ainsi réduire notablement la place de la subjectivité dans les décisions.

La question de la désaffiliation a été également discutée dans le cadre de la conception d'un tel outil. Dans les travaux qui mesurent l'appartenance aux gangs par l'auto-identification, les délinquants qui affirment ne plus faire partie d'un gang présentent une délinquance autorévélée moins importante que les délinquants qui se disent actuellement dans les gangs. Nombreuses sont les critiques qui ont été formulées concernant les efforts d'identification de la police et des bases de données spécifiquement destinées à identifier les délinquants membres de gangs de rue (Barrows et Huff, 2014). Par conséquent, toute démarche visant à documenter les processus d'affiliation doit s'accompagner d'une série de conditions permettant la désaffiliation. Le passage dans les gangs est temporaire pour plusieurs personnes contrevenantes (généralement une période allant d'une à deux années). Ne pas proposer de tels critères impliquerait invariablement une sur-identification du nombre de membres, avec toutes les conséquences que cela implique.

Dans le cadre de la présente démarche, différents critères seront déterminés afin de désigner les personnes qui ne doivent plus être considérées comme des membres de gangs de rue. Il va sans dire que les personnes qui ne sont plus actives d'aucune façon ou qui ne participent plus aux activités du gang ne devraient pas être considérées comme membres. Si l'identification est liée au fait d'atteindre certains seuils sur les principales dimensions désignées, il va sans dire que la désaffiliation suivra la même logique, à savoir que les personnes pour lesquelles les paramètres seront descendus en deçà de ces seuils devront être considérées comme d'anciens membres.

\section{Conclusion}

Pendant de nombreuses années, les organismes de prise en charge légale comme la police et les systèmes correctionnels ont eu recours à des stratégies typologiques d'identification des gangs et des membres de gangs. 
Le présent exercice propose l'idée d'un modèle de mesure des gangs et des membres inspiré du modèle multidimensionnel de Guay et Fredette (2010). En effet, ce modèle postule que les gangs ne sont pas des entités discrètes et l'exercice d'identification consiste à mesurer les différents groupes criminels et leurs membres sur des dimensions définitionnelles afin de déterminer des seuils au-delà desquels la réaction des organisations pénales devient utile, voire nécessaire. Bien qu'il ne permette pas de résoudre tous les problèmes liés à l'identification des membres, il offre une stratégie qui permet une mise à l'épreuve de la validité et de la fidélité. Il permet également d'étayer de manière plus empirique les règles d'identification et augmente ainsi la transparence de la démarche.

En somme, la définition des gangs et l'identification des membres est un exercice complexe aux retombées nombreuses, tant pour les organisations responsables de l'application de la loi que pour les personnes contrevenantes. Le processus d'identification des personnes contrevenantes associées aux gangs doit par conséquent se faire de manière à réduire les fâcheuses conséquences de la suridentification et de la sous-identification. Pour ce faire, elle doit reposer sur un processus encadré et transparent d'identification, basé sur les critères valides et fidèles. Cet article a permis de faire état des travaux d'un comité de travail qui met en commun l'expertise des intervenants des milieux de la recherche universitaire et du renseignement policier. Ce groupe a proposé une définition succincte du gang de rue et une infrastructure théorique pour en mesurer les différentes composantes permettant d'identifier les membres. Les travaux visant à tester l'accord interjuge des différents indicateurs sont présentement en cours, et des efforts pour estimer les propriétés psychométriques d'un tel instrument sont à venir. Une meilleure mesure de ce phénomène aux contours flous devrait permettre de mieux le cerner, et ainsi proposer des solutions pour en réduire ultimement les effets indésirables, tant par des efforts de prévention et de répression que de réinsertion.

\section{Références}

Anastasi, A. (1988). Psychological testing. New York, NY: Macmillan.

Barrows, J. et Huff, C. R. (2009). Les bases de données et les politiques publiques. Dans M. Le Blanc et M. Cusson (dir.), Traité de criminologie empirique (p. 281298). Montréal, Québec: Les Presses de l'Université de Montréal.

Bond, T. G. et Fox, C. M. (2001). Applying the Rasch model: fundamental measurement in the buman sciences. Mahwah, NJ: Lawrence Erlbaum Associates. 
Bureau of Justice Assistance. (1999). Urban street gang enforcement. Washington D.C: Government Printing Office.

Charland, M.-P. (2010). Les gangs de rue en prison (mémoire de maîtrise inédit). Université de Montréal, Québec, Canada.

Cohen, A. K. (1955). Delinquent Boys: The Culture of the Gang. Glencoe, IL: Free Press.

Corriveau, P. (2009). La violence dans l'univers des gangs: du besoin de protection à la construction identitaire masculine. Revue de l'Institut pour la prévention de la criminalité, 3, 117-134.

Cousineau, M.-M., Hamel, S. et Fournier, M. (2005). Les gangs du point de vue des jeunes, leur signification dans une trajectoire de vie. Dans N. Brunelle et M.-M. Cousineau (dir.), Trajectoires de délinquance juvénile. Les éclairages de la recherche qualitative (p. 97-119). Québec: Les Presses de 1'Université du Québec.

Cureton, S. R. (2002). Introducing Hoover: I'll ride for you, gangsta'. Dans C. R. Huff (dir.), Gangs in America III (p. 83-100). Thousand Oaks, CA: Sage Publications.

De Ayala, R. J. (2009). The Theory and Practice of Item Response Theory. New York, NY: The Guilford Press.

Decker, S. H. (1996). Collective and Normative Features of Gang Violence. Justice Quarterly, 13(2), 243-264.

Decker, S. H. et Pyrooz, D. C. (2010). Gang violence worldwide: Context, culture, and country. Dans Small Arms Survey 2010: Gangs, Groups, and Guns (p. 128-155). Cambridge, Royaume-Uni: Cambridge University Press.

Decker, S. H. et Van Winkle, B. (1996). Life in the Gang: Family, Friends, and Violence. New York, NY: Cambridge University Press.

DeLisi, M. (2003). Criminal careers behind bars. Behavioral Sciences and the Law, 21, 653-669.

Demers, A.-M. (2015). La délinquance et les gangs de rue selon la perspective des jeunes contrevenants: un regard qualitatif (Thèse de doctorat inédite). Université de Sherbrooke, Canada.

DeWalt, K. M. et DeWalt, B. R. (2002). Participant observation: a guide for fieldworkers. Walnut Creek, CA: AltaMira Press.

Dorais, M., et Corriveau, P. (2006). Jeunes filles sous influences: Prostitution juvénile et gangs de rue. Montréal, Québec: VLB Éditeur.

Egley, A. Jr. et Howell, J. C. (2013). Highlights of the 2011 National Youth Gang Survey. Washington, D.C.: U.S. Department of Justice, Office of Justice Programs, Office of Juvenile Justice and Delinquency Prevention.

Eisen, M. L. (2009). The Biasing Effect of Gang Evidence on Juror Decisions. Repéré à http://dx.doi.org/10.2139/ssrn.1436222

Esbensen, F.-A. et Huizinga, D. (1993). Gangs, drugs, and delinquency in a survey of urban youth. Criminology, 31, 565-589.

Esbensen, F.-A., Winfree, L. T., He, N. et Taylor, T. J. (2001). Youth Gangs and Definitional Issues: When is a Gang a Gang, and Why Does it Matter? Crime and delinquency, 47(1), 105-130. 
Felson, M. (2014). Les gangs et les guêpes. Dans J.-P. Guay et C. Fredette (dir.), Le phénomène des gangs de rue: théories, évaluations, interventions (p. 115-136). Montréal, Québec: Les Presses de l'Université de Montréal.

Fredette, C. (2014). Mesurer l'apport unique de l'adbésion à la culture des gangs de rue à l'explication des conduites délinquantes (Thèse de doctorat inédite). Université de Montréal, Canada.

Gaes, G. G., Wallace, S., Gillman, E., Klein-Saffran, J. et Suppa, S., (2002). The influence of prison gang affiliation on violence and other prison misconduct. The Prison Journal, 82, 359-385.

Gatti, U., Tremblay, R. E., Vitaro, F. et McDuff, P. (2005). Youth gangs, delinquency and drug use: a test of the selection, facilitation, and enhancement hypotheses. Journal of Child Psychology and Psychiatry, 46, 1178-1190.

Guay, J.-P. (2012). Predicting recidivism with street gang members. Ottawa, Ontario: Public Safety Canada.

Guay, J.-P. et Fredette, C. (2010). Le phénomène des gangs de rue et la mesure. Dans M. Le Blanc et M. Cusson (dir.), Traité de criminologie empirique (p. 167198), Montréal, Québec: Les Presses de l'Université de Montréal.

Guay, J.-P., Fredette, C. et Dubois, S. (2014). Définir, classifier et mesurer les gangs de rue et leurs membres. Dans J.-P.Guay et C. Fredette (dir.), Le phénomène des gangs de rue: théories, évaluations, interventions (p. 17-36). Montréal, Québec: Les Presses de l'Université de Montréal.

Guay, J.-P. et Gaumont-Casias, J. (2009). Sur la mesure du phénomène des gangs de rue au Québec: vers le développement d'un cadre de mesure. Québec: Gouvernement du Québec, Ministère de la Sécurité publique, Direction de la recherche.

Hagedorn, J. M. (2008). A World of Gangs: Armed Young Men and Gangsta Culture. Minneapolis, $\mathrm{MN}$ : University of Minnesota Press.

Huff, C. R. (1989). Youth Gangs and Public Policy. Crime er Delinquency, 35(4), 524-537.

Hughes, L. A. et Short, J. F. Jr. (2005). Disputes Involving Youth Street Gang Members: Micro-Social Contexts. Criminology, 43(1), 43-76.

Katz, C. M. (2003). Issues in the Production and Dissemination of Gang Statistics: An Ethnographic Study of a Large Midwestern Police Gang Unit. Crime and Delinquency, 49(3), 485-516.

Katz, C. M. et Webb, V. J. (2006). Policing Gangs in America. Cambridge, Royaume-Uni: Cambridge University Press.

Klein, M. W. (1995). The American Street Gang: Its nature, prevalence, and control. Oxford, Royaume-Uni: Oxford University Press.

Klein, M. W. et Maxson, C. L (2006). Street Gangs: Patterns and Policies. Oxford, Royaume-Uni: Oxford University Press.

Krohn, M. D. et Thornberry, T. P. (2008). Longitudinal Perspectives on Adolescent Street Gangs. Dans A. M. Liberman (dir.), The Long View of Crime: A Synthesis of Longitudinal Research (p. 128-160). New York, NY: Springer Sciences and Business Media. 
Lanctôt, N. et LeBlanc, M. (1997). Les adolescentes de bandes marginales: un potentiel antisocial atténué par la dynamique de la bande? Criminologie, 30(1), 111-130.

Landre, R., Miller, M. et Porter, D. (1997). Gang: A Handbook for Community Awareness. New York, NY: Facts on File Inc.

Lawshe, C. H. (1975). A quantitative approach to content validity. Personnel Psychology, 28, 563-575.

Lynn, M. R. (1986). Determination and quantification of content validity. Nursing Research, 35, 382-385.

McGloin, J. M. (2005). Policy and Intervention Considerations of a Network Analysis of Street Gangs. Criminology and Public Policy, 4(3), 607-635.

McShane, M. D., Williams, F. P. et Dolny, H. M. (2003). Effect of Gang Membership on Parole Outcome. Journal of Gang Research, 10, 25-38.

Melde, C. et Esbensen, F.-A. (2011). Gang membership as a turning point in the life course. Criminology, 49, 513-552.

Miethe, T. D. et McCorkle, R. C. (1997). Gang membership and Criminal Processing: A test of the "Master Status" concept. Justice Quarterly, 14, 407-427.

Morselli, C. (2009). Inside Criminal Networks. New York, NY: Springer.

Papachristos, A. V. (2006). Social Network Analysis and Gang Research: Theory and Methods. Dans J. F. Short et L. A. Hugues (dir.), Studying Youth Gangs (p. 99-116). Lanham, MD: AltaMira.

Sachs, J. (1997). Street Gang Awareness: A Resource Guide for Parents and Professionals. Minneapolis, MN: Fairview Press.

Sanchez-Jankowski, M. (1991). Islands in the Street: Gangs and American Urban Society. Berkeley, CA: University of California Press.

Sanders, W. B. (1994). Gangbangs and Drive-Bys: Grounded Culture and Juvenile Gang Violence. New York, NY: Aldine De Gruyter.

Sarnecki, J. et Petterson, T. (2001). Criminal Networks in Stockholm. Dans M. W. Klein, H.-J. Kerner, C. L. Maxson et E. G. M. Weitekamp (dir.), The Eurogang Paradox: Street Gangs and Youth Groups in the U.S. and Europe (p. 257272). Dordrecht, Pays-Bas: Kluwer Academic Publishers.

Savelli, L. (2004). Gangs across America and Their Symbols. New York, NY: Looseleaf Law Publications Inc.

Spergel, I. A. (1995). The Youth Gang Problem: A Community Approach. Oxford, Royaume-Uni: Oxford University Press.

Thornberry, T. P., Krohn, M. D., Lizotte, A. J., Smith, C. A. et Tobin, K. (2003). Gangs and Delinquency in Developmental Perspective. New York, NY: Cambridge University Press.

Thrasher, F. M. (1927). The Gang: A Study of 1313 Gangs in Chicago. Chicago, IL: University of Chicago Press.

Totten, M. D. (2000). Guys, Gangs E Girlfriend Abuse. Peterborough, Ontario: Broadview Press.

Vigil, J. D. (1988). Barrio Gangs: Street Life and Identity in Southern California. Austin, TX: University of Texas Press. 
Weerman, F. M., Lovegrove, P. J. et Thornberry, T. (2015). Gang membership transitions and its consequences: Exploring changes related to joining and leaving gangs in two countries. European Journal of Criminology, 12, 70-91.

Wilson, M. (2005). Constructing Measures: An Item Response Modeling Approach. Mahwah, NJ: Erlbaum.

Wortley, S. (2010). Identifier les gangs de rue: Dilemmes définitionnels et répercussions sur les politiques. Ottawa, Ontario: Sécurité publique Canada.

Yablonsky, L. (1970). The Violent Gang. Chicago, IL: University of Chicago Press.

\section{Defining Street Gangs and Identifying Street Gang Members for Police Purposes}

ABSTRACT - Defining what constitutes a street gang and how to identify gang members is a complex endeavour. A number of things characteristic of these groups, notably their elusive and clandestine nature, their heterogeneity, and their continually changing features, have led us to modify our conceptualizations. This article proposes a simple general definition that describes the street gang as an organized criminal group distinguished from those organized crime groups that are defined by law 467.1. Emphasis is placed on the operational nature of markers of association and the rationale behind their development. A new definition of these gangs, combined with an operationalization based on criteria observed by police organizations, offers multiple advantages over definitions developed within an academic setting. The theoretical implications and practical issues of the new definition are also addressed.

KEYWORDS - Street gangs, definition, identification, organized crime, police.

\section{Definición de las pandillas callejeras y identificación de sus miembros con propósitos policiales}

RESUMEN - El ejercicio que busca definir a las pandillas callejeras e identificar a sus miembros es complejo. Varias características de esos grupos, especialmente sus caracteres de evasión y clandestinidad, su gran heterogeneidad, así como su mutabilidad nos han llevado a modificar nuestra conceptualización sobre las pandillas. El presente artículo propone una definición general y simple que describe a las pandillas callejeras como un grupo criminal organizado que se distingue de los otros grupos del crimen organizado definidos por la ley 467.1. Sin embargo, se hace hincapié en el carácter operativo de las marcas de pertenencia y de la lógica que subyace en su desarrollo. Una nueva definición de las pandillas callejeras, acompañada de una operacionalización basada en criterios observables por las organizaciones policiales y por los organismos que operan en el ámbito penal, ofrece varias ventajas en comparación a una definición desarrollada en un marco académico. Se abordan también las implicaciones teóricas y las cuestiones teóricas.

PALABRAS CLAVE - Pandillas callejeras, definición, identificación, delincuencia organizada, policía. 